\title{
COORDINATED CONTROL FOR VEHICLES COOPEARATIVE MANEUVERS USING DISTRIBUTED MODEL PREDICTIVE CONTROL.
}

\author{
ANDONI URUTEGUI \\ Industry \& Transportation, TECNALIA Research \& Innovation \\ Derio, Spain \\ RAY LATTARULO \\ Industry \& Transportation, TECNALIA Research \& Innovation \\ Derio, Spain \\ GERARDO FERNÁNDEZ-LÓPEZ \\ Mechatronic Group, Simón Bolívar University \\ Sartenejas, Venezuela
}

\begin{abstract}
The research and development focused towards autonomous vehicles has grown quickly and progressively, in search of a more efficient, safe and clean future. Thus, is necessary to consider: cooperation between multiple ( 2 or more) vehicles, security measures, priorities, among others. Under this framework we present a design of predictive controller for the management of cooperative maneuvers, considering safety, efficiency and comfort in driving, as well as a series of physical limitations of the vehicle, all this to obtain a speed profile that guarantees the optimal execution (locally) of the maneuver.
\end{abstract}

\section{Introduction}

Recent research suggest that Intelligent Transportation Systems aim to be the standard mode of transportation in the future, and for that to be possible we need to focus the investigation on real world scenarios, taking into consideration the complexity of multi-vehicle transit and its multiple variables. Within that framework we propose a solution to the problem where the vehicles must execute cooperative maneuvers. Several lines of research consider predictive control strategies for the resolution of this type of problems, because of the advantages that it presents in terms of handling constraints. Li et al [1] propose a trajectory tracking of mobile robots. [2] shows how MPC handles the steering of mobile robots in conjunction with visual processing. Recent works like the ones of Riegger et al [3] and Nordell [4] present centralized solutions for vehicle intersections and overtaking maneuvers. In this work a predictive control system is used in a distributed manner, similar to [5], where each agent has an MPC that contributes to the generation of a speed profile that allows the successful execution of the maneuver. The methodology of modeling the maneuvers inside the predictive controller is introduced as a novelty, meaning that is the maneuver state as a whole what is calculated at each instant. 


\section{Modeling}

For simplicity reasons, the following considerations are taken: (1) scenarios are design with two autonomous vehicles, (2) the vehicle trajectories are defined, (3) fully working vehicle to vehicle communication is present, (4) the information shared at time $\mathrm{t}$ is available to the other vehicle at time $\mathrm{t}+\mathrm{k}$. The point-mass model in Eq. (1) will be used.

$$
\begin{gathered}
\dot{x}=v \\
\dot{v}=a \\
\dot{a}=J r
\end{gathered}
$$

\subsection{Intersection maneuver}

The aforementioned model is manipulated to generate a system of differential equations that describe the maneuver, first, the $\mathrm{X}$ distance between vehicles through the point of the intersection (collision point) is calculated.

$$
X=d_{1}+d_{2}
$$

Expanding the point-mass model, a system of relative states is found in Eq. (3), where $\mathrm{X}$ is the distance between vehicles, $\mathrm{V}$ is the relative velocity and $\mathrm{A}$ the relative acceleration. This model is used for the state predictions, treating the maneuver state as a whole.

$$
\begin{aligned}
& \dot{X}=v_{1}+v_{2} \\
& \dot{V}=a_{1}+a_{2} \\
& \dot{A}=J r_{1}+J r_{2}
\end{aligned}
$$

\subsection{Obstacle avoidance.}

The vehicles are in opposite lanes of the road and one of them must occupy the lane against-flow momentarily to continue on its way. The X distance is calculated considering a safety barrier, as proposed in [6], placed at a distance that depends on the maneuver speeds, initially at $3 \mathrm{~L}$ of the, where $\mathrm{L}$ is the length of vehicle 1 .

\subsection{Maneuver parameters}

The parameters represent the MPCs variable constraints; First of all, we must ensure that the vehicles do not collide, maintaining at least a minimum safety distance between them:

$$
X_{(t+k \mid t)}>X_{\text {safe }(t+k \mid t)}
$$

$(t+k \mid t)$ being the predicted instant $t+k$ calculated in $t$ by the MPC using Eq.3 and previous values as inputs. Additionally, for efficiency reasons we want this to happen as close as possible to a reference speed: 


$$
0<v_{1(t+k \mid t)} \leq v_{r e f(}(t+k \mid t)
$$

Velocity constraint written in terms of $\mathrm{V}$ :

$$
v_{2(t+k \mid t)}<V_{(t+k \mid t)}<v_{r e f}(t+k \mid t)+v_{2(t+k \mid t)}
$$

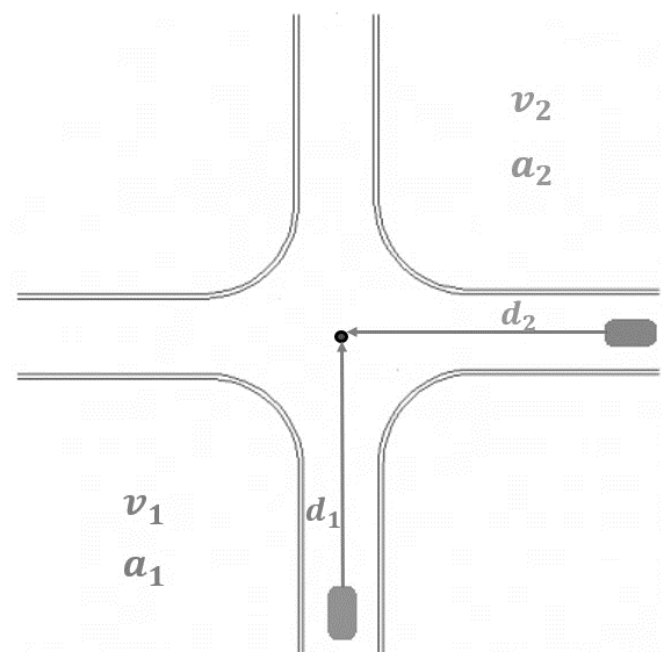

Figure 1. Intersection maneuver

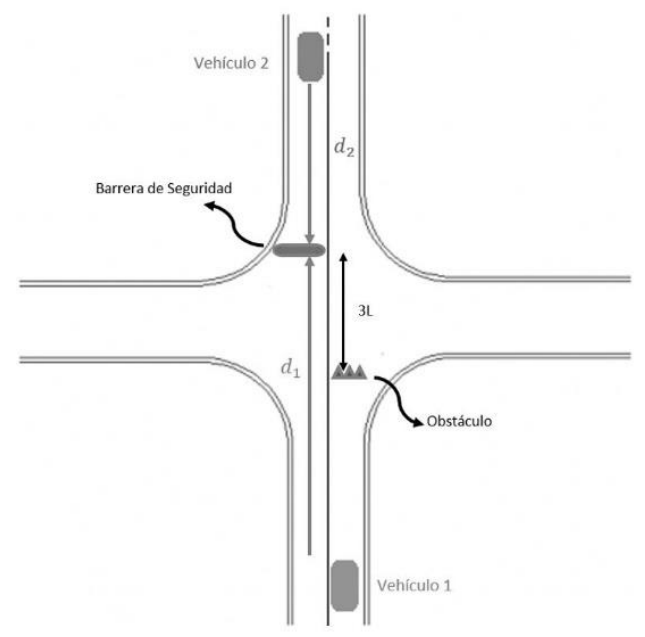

Figure 2. Obstacle Avoidance maneuver 
Finally, considering driving comfort, violent accelerations and decelerations are limited, resulting in the following constraint:

$$
a_{\min (t+k \mid t)}+a_{2}<A_{(t+k \mid t)}<a_{\operatorname{máx}(t+k \mid t)}+a_{2}
$$

\section{Predictive controller}

The function of the MPC is to obtain a control signal, ensuring that all the conditions are satisfied and that the system variables remain within their limits. It is proposed: (1) the MPC Prediction Horizon, (2) the cost function for optimization, and (3) the control input $u_{(t)}$ of the system.

At the top speed of $20 \mathrm{Km} / \mathrm{h}$ the test vehicle, a Renault Twizy, takes 14 meters to stop, a horizon of 10 samples at $300 \mathrm{~ms}$ is chosen, thus, making predictions for the next 17 meters of travel, enough for an emergency stop. The cost function has the objective of minimizing the mean square error of the current speed with its reference:

$$
J=\sum_{t=0}^{10}\left(v_{r e f}(t+k \mid t)-v_{(t+k \mid t)}\right)^{2}
$$

The jerk is chosen as control variable, with the only purpose of taking comfort into consideration, forcing the system to make its predictions within comfortable jerk values. This system is proposed as a high-level controller and its output is not used for further stages, the MPC predictions are instead, they guarantee that the predicted values of $\mathrm{V}$ comply with the maneuver requirements, so they are used as a reference for the lower level controllers as a maneuver speed profile. Generally, this type of MPC is applied as a centralized maneuver solving unit, where every agent sends its information and then receive the speed profile. In this case each vehicle has an MPC, at each timestep every vehicle shares it information to the other so that the optimization problem can be solved locally, reducing the computational load on the computer and making the system more robust in case one vehicle malfunctions. Additionally, if each vehicle is programmed to meet the condition of safety distance, it is necessary to implement priorities. This decision system is based on the calculations of reaction distance and breaking distance proposed in [7] [8], reducing it to the point of granting a higher priority (the value of zero) to the vehicle that has less reaction time.

\section{Results}

The vehicles are set to collide, giving priority to vehicle 1 , which results in the lifting of its MPC constraints. The MPC on vehicle 2 predicts that a safety violation will occur and generates a speed profile that reduces velocity as shown by the blue curve in Figure 3. 


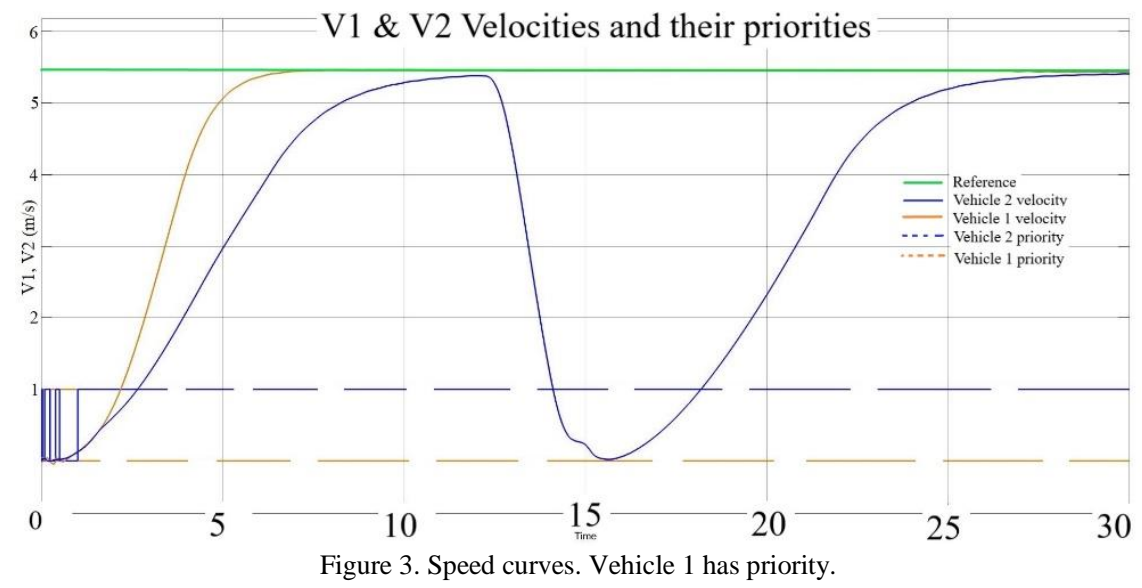

Safety distance was modified to values of $10 \mathrm{~m}, 20 \mathrm{~m}$ and $40 \mathrm{~m}$, the curves shown in Figure 4 demonstrate how the controller executes the corrective actions earlier in time while the safety distance is increased. Both curves stabilize at 5.5 $\mathrm{m} / \mathrm{s}$ which corresponds to the reference value, meaning that the optimization of the cost function is done correctly. Also, is shown in both figures that the variables are within their bounds at all times, meaning that the constraints are being respected by the MPC calculations.

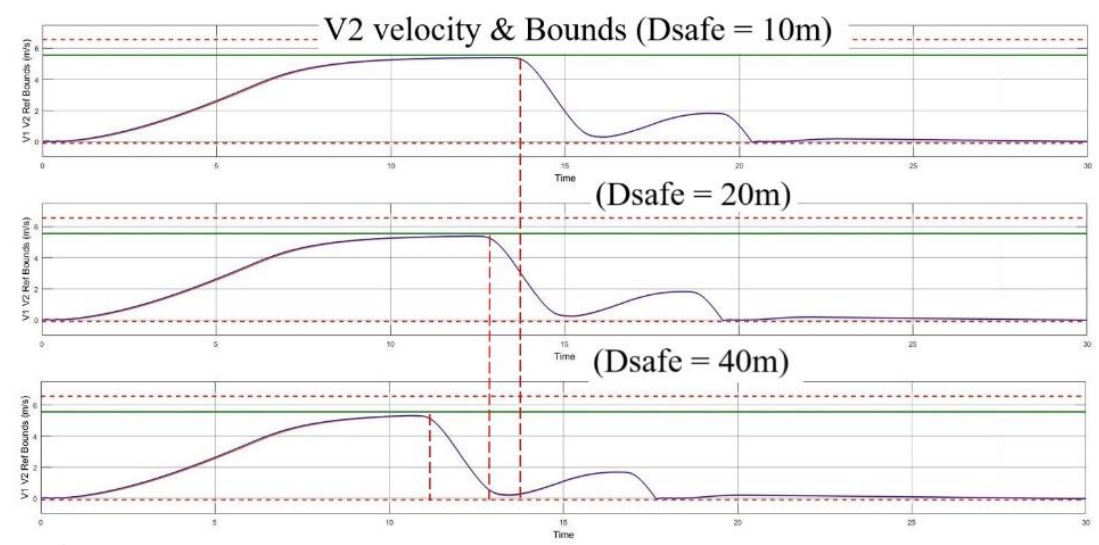

Figure 4. Speed curves at different safety distance values.

\section{Conclusions and future works}

Predictive controllers, together with the other modern control strategies, present promising results in terms of control systems, showing the advantage they have 
over other strategies. Its relative ease of modeling in MIMO systems, the robustness of applying corrections to future errors and the distributed approach are essential characteristics for a real-time application such as autonomous vehicles. Regarding future work, an extension of its scope is considered, that is, the design of a controller that involves maneuvers of more than two vehicles, the inclusion of external agents in the cost function and, in general terms, search for the application of this strategy in all maneuvers that the vehicle can perform.

\section{References}

1. Z. Li, J. Deng, R. Lu, Y. Xu, J. Bai and C. Su, "Trajectory-Tracking Control of Mobile Robot Systems Incorporating Neural-Dynamic Optimized Model Predictive Approach," in IEEE Transactions on Systems, Man, and Cybernetics: Systems, vol. 46, no. 6, pp. 740-749, June 2016.

2. Z. Li, C. Yang, C. Su, J. Deng and W. Zhang, "Vision-Based Model Predictive Control for Steering of a Nonholonomic Mobile Robot," in IEEE Transactions on Control Systems Technology, vol. 24, no. 2, pp. 553-564, March 2016.

3. Riegger, L., Carlander, M., Lidander, N., Murgovski, N., \& Sjöberg, J. (2016, November). Centralized mpc for autonomous intersection crossing. In Intelligent Transportation Systems (ITSC), 2016 IEEE 19th International Conference on (pp. 1372-1377). IEEE.

4. Nordell, B. (2016). Trajectory Planning for Autonomous Vehicles and Cooperative Driving.

5. Alexander Katriniok, Peter Kleibaum, and Martina Jo`sevski. (2017). Distributed model predictive control for intersection automation using a parallelized optimization approach. IFAC-PapersOnLine, 50(1):59405946.

6. Joshué Pérez Rastelli. (2012). Agentes de control de vehículos autónomos en entornos urbanos y autovías. (Agents control for autonomous vehicles in urban and highways environments). $\mathrm{PhD}$ thesis, Complutense University of Madrid, Spain.

7. P, Delaigue and A, Eskandarian. (2014) A comprehensive vehicle braking model for predictions of stopping distances. Proceedings of the Institution of Mechanical Engineers, Part D: Journal of Automobile Engineering, 218(12):1409-1417.

8. Yuan-Lin Chen and Chong-An Wang. (2017) Vehicle safety distance warning system: A novel algorithm for vehicle safety distance calculating between moving cars. In Vehicular Technology Conference, 2007. VTC2007-Spring. IEEE 65th, pages 2570-2574. IEEE. 